\title{
Cell sheet Transplantation in the Treatment of Fetal Myelomeningocele
}

Ubaidus Sobhan $^{1,2}$, Rie Irie ${ }^{3}$, Tatsuzo Hebiguchi ${ }^{2}$, Hiromasa Yamashita ${ }^{2}$, Yasuhiko Tabata ${ }^{4}$ and Toshio Chiba ${ }^{2,3,5^{*}}$

${ }^{1}$ Tokyo Dental College, Department of Physiology, Tokyo, Japan

${ }^{2}$ Kairos Co. Ltd, R\& D Department, Tokyo, Japan

${ }^{3}$ Department of Pediatric Surgery, Showa University, Tokyo, Japan

${ }^{4}$ Institute for Frontier Medical Science, Kyoto University, Kyoto, Japan

${ }^{5}$ Waseda University, Faculty of Science and Engineering, Tokyo, Japan

*Corresponding author: Toshio Chiba, Kairos Co. Itd, R\&D Department, 2-3-16, Kanda, Surugadai, Chiyoda-Ku, Tokyo, 101-0062, Japan, Tel: +81-3-5577-6829; Fax: +81-3-5577-6839; E-mail: chiba@kairos-8k.co.jp

Rec date: October 24, 2017; Acc date: November 3, 2017; Pub date: November 8, 2107

Copyright: (C) 2017 Chiba T, et al. This is an open-access article distributed under the terms of the Creative Commons Attribution License, which permits unrestricted use, distribution, and reproduction in any medium, provided the original author and source are credited

\begin{abstract}
Myelomeningocele (MMC) is a devastating anomaly with lifelong disability and morbidity. This is the first nonlethal disease as an indication of possible fetal surgery, and currently is the most common disease having undergone open fetal surgery. Although fetal surgical repair alone has been partially improving postnatal long-term clinical outcome, additional innovative strategy to be coupled with should be further investigated. In this regard, we studied the significance of tissue engineering technology as a means of provoking synergistic regeneration of the spinal cord that has been injured due to the associated MMC by the time of fetal anatomical repair. The aim of this report is to evaluate therapeutic feasibility of prenatal direct coverage of the MMC using an adherent sheet-like cluster of cultured myoblasts, the cell sheet. In our study using SD rat as a retinoic-acid-induced model of fetal MMC, a piece of the sheet was directly attached onto the surface of the MMC lesion following anesthesia, laparotomy and hysterotomy. As a result, we could reveal that the cell sheet could histologically seal up the lesion if it was properly kept stuck 4, 6 and 24 hours in utero. In conclusion, the cell sheet, as a part of therapeutic strategy, is likely to work for improving the outcome of correcting fetal MMC surgically.
\end{abstract}

Keywords: Myelomeningocele; Open fetal surgery; Retinoic-acidinduced MMC; Cell sheet technology

Abbreviations: CST: Cell Sheet Transplantation; MMC: Myelomeningocele; NGF: Nerve Growth Factor; ONTD: Open Neural Tube Defect; SMA: Smooth Muscle Actin; SC: Spinal Cord

\section{Introduction}

Myelomeningocle (MMC) is a most common type of open neural tube defect (ONTD). Usually, MMC is a non-fatal but neurologically devastating congenital anomaly that leaves affected children with lifelong motor paralysis of the lower extremities, skeletal deformities, urinary and fecal incontinence, sexual dysfunction, and cognitive disabilities [1-4]. Surgical correction of fetal MMC has been predominantly performed to suppress the progression of in utero histological damages of the exposed spinal cord expecting clinical amelioration of postnatal neurological dysfunction although it could reportedly be associated with several fetomaternal risks including preterm birth with prematurity. In order to further improve the therapeutic outcome, we attempted to synergistically provoke regeneration of the spinal cord that had been injured by the time of fetal surgical correction. For this purpose, we experimentally adopted an adherent sheet of cultured cells (a "cell sheet"), which could be expected to histologically seal up the MMC lesion in utero. The cell sheet-based tissue engineering has been successfully applied in the field of diverse regenerative medical fields (myocardium, cornea, epithelium, esophagus, lung, liver, pancreas, thyroid, and periodontal tissue) [5,6]. Experimentally, we assessed the biological effects of direct coverage of the MMC lesion on regeneration of the injured spinal cord using SD rat fetuses having retinoic-acid-induced fetal MMC. We found that the cell sheet could histologically seal up the lesion if it was properly kept stuck in utero for a given period of time on the surface. This study is the first to use the cell sheet technology as a supplemental strategy to be coupled with the current open fetal surgical procedure for fetal MMC. Using the cell sheet directly sealing the MMC lesion, we report the outcome of histological and immunohistochemical studies as well as its potential effects on regeneration of the injured spinal cord.

\section{Materials and Methods}

\section{Study design for cell sheet treatment of fetal myelomeningocele}

All animals were treated in accordance with the guiding principles for the care and use of animals in Tokyo Dental College (Tokyo, Japan). Time-dated (embryonic day 10) pregnant Sprague-Dawley (SD) rats were gavage fed with $60 \mathrm{mg} / \mathrm{kg} /$ body weight of retinoic acid (Wako, Japan) that were dissolved in olive oil. Rat fetuses, which had retinoic acid-induced MMC and underwent in utero cell sheet treatment (embryonic day 10) were harvested (embryonic day 19) along with control animals.

\section{Cell culture and preparation of cell sheet}

L6 cells, a myoblast cell line obtained from rat skeletal muscle, were purchased from American Type Culture Collection (ATTC Rockville, $\mathrm{MD}$ ) and were grown in $10 \mathrm{~cm}$ plates to near confluence. Rat L6 cell 
lines were cultured on $25 \mathrm{~cm}$ tissue culture dish (BD Biosciences, Franklin Lakes, NJ, USA) with DMEM (Gibco, Brooklyn, NY, USA) supplemented with $10 \%$ fetal bovine serum (FBS; Hyclone, Shrewsbury, NJ, USA), $100 \mathrm{IU} / \mathrm{ml}$ penicillin and $100 \mu \mathrm{g} / \mathrm{ml}$ streptomycin (Gibco). The cell sheet technology mainly depends on the use of a specific thermo-responsive cell culture dish ("cell seed"), which enables to make a sheet-like cluster of cultured cells ("cell sheet"). For our experiment, L6 myoblast cells were transferred into the "cell seed" and were cultured until the cells became confluent and the sheet could reversibly adhere to and detach from the dish by thermally controlled surface hydrophobicity [5].

\section{Cell sheet transplantation}

Cell sheet was transplanted on retinoic acid - induced MMC fetuses on embryonic day 19 . Then, a small incision was made with a 6-0-silk purse-string suture put on the uterine wall. The amniotic sac was cut open and the spinal cord having MMC was exposed. Cell sheet was placed onto the MMC lesion. The uterus was reopened 4, 6 and 24 hours after the cell sheet transplantation and the MMC lesions where the transplanted cell sheet was remaining stuck were studied immunohistochemically.

\section{Histological study and immunohistochemistry}

The entire fetus that underwent cell sheet transplantation was fixed in $10 \%$ neutral buffered formalin (Sigma-Aldrich) immediately after delivery. After histoprocessing, cell sheet and the CST specimens were embedded in paraffin wax and sectioned at $5 \mu \mathrm{m}$ thickness. Sections were serially dewaxed, rehydrated, and stained with hematoxylin and eosin. To evaluate the differentiation potential of those myoblast cells transplanted onto the spinal column, sections were analyzed by immunostaining using immunofluorescent antibodies against neuron, skeletal muscle and myoblast cell markers [7]. These sections were incubated with primary antibodies including rabbit polyclonal AntiNGF and mouse monoclonal Anti-SMA (Abcam, Cambridge, UK; dilution 1:100). Nerve growth factor (NGF), a member of the NGFbeta family is a transiently expressed specific marker for developing glial cells and neuroepithelial stem/progenitor cells in developing CNS. As NGF is involved in regulation of growth and differentiation of sympathetic and certain sensory neurons, it's down regulation correlates with differentiation of these cells. The expression of smooth muscle alpha actin (SMA) is relatively restricted to vascular smooth muscle cells regulating their myogenetic proliferation, repair and regeneration which could be affected by pathological conditions including cell transformation. Finally, these immunostained sections were evaluated using a conventional fluorescence microscope (Zeiss, Jena, Germany).

\section{Results}

\section{Histology and immunostaining of cell, cell sheet and the CST specimens}

The cultured L6 myoblast cell line displayed fibroblast-like, spindleshaped morphology. No obvious morphological changes were observed during the culture period. Figure a shows the cell sheet in a specific culture dish. In figure b, H-E staining of the cell sheet shows the cells are attached to each other. Figures $c$ and d show the MMC lesion along with the transplanted cell sheet. In figures e and $f$, the immunostaining microscopic picture of cultured L6 cells and the cell sheet show positive staining of both the SMA, and NGF. Likewise, in figures $\mathrm{g}, \mathrm{h}, \mathrm{j} \mathrm{k}, \mathrm{l}$, and I the MMC lesions of the three experimental groups of fetuses (4, 6 and 24 hours after the cell sheet transplantation) on which the cell sheet was remaining attached showed positive staining of both the SMA and NGF.

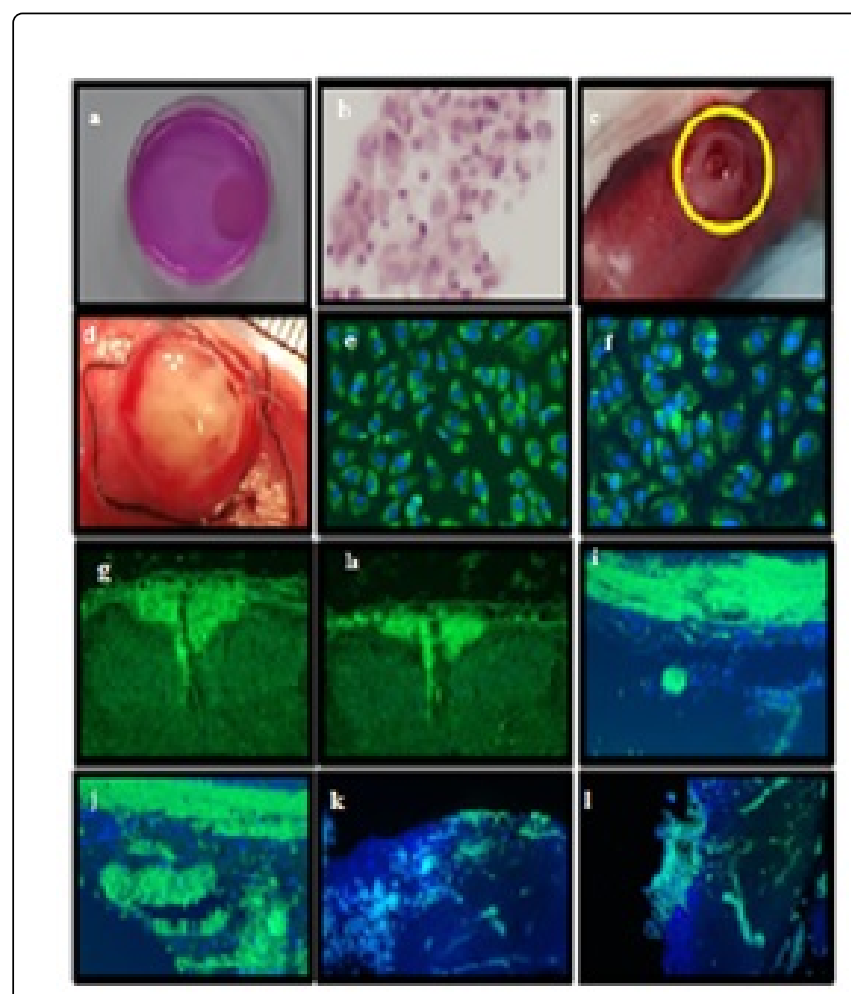

Figure 1: Figure a shows the cell sheet on the special dish. H-E staining of cell sheet shows in figure b. Figure $\mathrm{c}$ shows the retinoic acid induced MMC model fetus at E-19 before the CS transplantation. Figure $\mathrm{d}$ shows the CST on the MMC defective area. Figure $\mathrm{e}$ and $\mathrm{f}$ shows the immunostaining of SMA, NGF positive cultured L6 myoblast cells. Figures i, j, k, l, show the immunopositive reaction of SMA and NGF on the transplanted area of defective spinal cord after 4, 6 and 24 hours.

\section{Discussion}

The outcome of our study is quite encouraging because it suggests that a cell sheet directly engrafted onto the fetal MMC can potentially stimulate regeneration of the injured neural tissue. Prenatal correction of the MMC for prevention of cerebrospinal fluid leakage that is likely to result in hydrocephalus and an attenuation of progression of cord damages in utero [3] has obviously improved the postnatal neurological function with markedly reduced lifelong morbidity. As such, open fetal surgery combined with simultaneous cell sheet coverage of the MMC will potentially help achieve prenatal care synergistically with concomitant stimulation of regenerative recovery of the cord injured before the fetal anatomical correction [8].

We employed the cell sheet technology because this was quite simple and preferable, i.e., just a simple temperature change can alter the surface characteristics of the specifically designed culture dishes allowing the attached sheet of cell clusters to detach with the cell membrane connections and cell-to-cell communications preserved 
Citation: Sobhan U, Irie R, Hebiguchi T, Yamashita H, Tabata Y, et al. (2017) Cell sheet Transplantation in the Treatment of Fetal Myelomeningocele. Gen Med (Los Angeles) 5: 303. doi:10.4172/2327-5146.1000303

Page 3 of 3

well. We also could expect that the use of the cell sheet technology is more advantageous compared with other cell delivery modality in light of a higher cell density as well as a highly efficient regenerative potential. Actually, the cell sheet-based tissue engineering has been medically accepted and successfully applied in the field of regeneration of pathologically affected myocardium, cornea, epithelium, esophagus, lung, liver, pancreas, thyroid, and periodontal tissues [4,9]. Naturally, cell sheet technology might work better in the fetal biological environment where highly proliferative stem cells are actively working in the amniotic fluid as well as in a variety of fetal tissues. Furthermore, cell division is obviously supposed to be more activated prenatally than in the adulthood with potentially faster tissue growth. In our experiment, the cell sheet was engrafted and transplanted onto the retinoic acid-induced fetal rat MMCs at the embryonic 19 days. Importantly enough, when the uterus was reopened 4, 6 and 24 hours after, we found that the sheet was staying adhered and histologically attached to the MMC, i.e., an immunohistochemically positive neuron marker was demonstrated to be expressed suggesting probable regeneration of the nerve tissue (Table 1).

\begin{tabular}{|l|l|l|l|}
\hline Experiments & Total Animals & Total fetus & CST Fetus \\
\hline 4 hours & 6 & 65 & 6 \\
\hline 6 hours & 4 & 49 & 4 \\
\hline 24 hours & 8 & 78 & 8 \\
\hline \multicolumn{2}{|l}{ CST- Cell sheet transplantation }
\end{tabular}

Table 1: This table shows the total animals, total fetus and the cell sheet transplanted fetus in 3 groups.

In this study, we investigated the likelihood of neural and muscle tissue regeneration with the use of the cell sheet for up to $24 \mathrm{hrs}$ after its surgical transplantation. The outcome of our study could be suggestive of neuronal regeneration stimulated by fetal cell sheet transplantation based on the positive immunohistological staining of NGF and SMA.

In conclusion, fetal MMC management by a cell sheet transplantation coupled with anatomical correction could stimulate more its histological and functional recovery than fetal surgical correction alone. Accordingly, the cell sheet technology could be expected to alter the management strategy of fetal MMCs from an early-stage fetal surgical correction just to prevent in utero progression of spinal cord damage to a combined employment of the cell sheet technology for synergistic regeneration of the injured spinal cord.

\section{Funding}

No current external funding sources for this study.

\section{Ethical Approval}

This article does not contain any studies with human participants performed by any of the authors.

\section{Conflict of Interest}

The authors declare that they have no conflict of interest.

\section{References}

1. Adzick NS (2013) Fetal surgery for spina bifida: Past, present, future. Semin Pediatr Surg 22: 10-7.

2. Adzick NS (2012) Fetal surgery for myelomeningocele: Trials and tribulations. Isabella Forshall Lecture. J Pediatr Surg 47: 273-281.

3. Danzer E, Johnson MP, Adzick NS (2012) Fetal surgery for myelomeningocele: Progress and perspectives. Dev Med Child Neurol 54: 8-14.

4. Sutton LN (2008) Fetal surgery for neural tube defects. Best Pract Res Clin Obstet Gynaecol 22: 175-188.

5. Yang J, Yamato M, Shimizu T, Sekine H, Ohashi K, et al. (2007) Reconstruction of functional tissue with cell sheet engineering. Biomaterials 28: 5033-5043.

6. Matsuura K, Utoh R, Nagase K,Okano T (2014) Cell sheet approach for tissue engineering and regenerative medicine. J Control Release 190: 228-239.

7. Sobhan U, Sato M, Shinomiya T, Okubo M, Tsumura M, et al. (2013) Immunolocalization and distribution of functional temperature-sensitive TRP channels in salivary glands. Cell Tissue Res 354: 507-519.

8. Vrecenak JD, Flake AW (2013) Fetal surgical intervention: Progress and perspectives. Pediatr Surg Int 29: 407-417.

9. Sawa Y, Miyagawa S, Sakaguchi T, Fujita T, Matsuyama A, et al. (2012) Tissue-engineered myoblast sheets improved cardiac function sufficiently to dis-continue LVAS in a patient with DCM: Report of a case. Surg Today 42: 181-184. 